\title{
IMPROVED SHOCK-CAPTURING OF JAMESON'S SCHEME FOR THE EULER EQUATIONS
}

\author{
J. W. VAN DER BURG, J. G. M. KUERTEN AND P. J. ZANDBERGEN \\ Faculty of Applied Mathematics, University of Twente, Postbox 217, 7500 AE Enschede, The Netherlands
}

\begin{abstract}
SUMMARY
It is known that Jameson's scheme is a pseudo-second-order-accurate scheme for solving discrete.conservation laws. The scheme contains a non-linear artificial dissipative flux which is designed to capture shocks. In this paper, it is shown that the. shock-capturing of Jameson's scheme for the Euler equations can be improved by replacing the Lax-Friedrichs' type of dissipative flux with Roe's dissipative flux. This replacement is at a moderate expense of the calculation time.
\end{abstract}

KEY wORDS Multigrid Runge-Kutta Conservation laws

\section{INTRODUCTION}

The development of second-order-accurate high-resolution schemes for compressible flows is a major topic in computational fluid dynamics (CFD). First-order upwind schemes such as Roe's ${ }^{1}$ scheme have the ability of resolving grid-aligned shocks accurately. However, first-order-accurate schemes are generally considered too dissipative in the smooth part of the solution. For computing the steady-state solution of the Euler equations, Jameson ${ }^{2}$ constructed a pseudosecond-order-accurate scheme by adding artificial dissipation to a central flux difference scheme. Jameson used a Lax-Friedrichs' type of dissipative flux in order to capture shocks and a fourthorder term to provide a base level of dissipation in smooth parts of the solution. In order to obtain the steady-state solution, Jameson employed Runge-Kutta time-stepping with frozen dissipation. Convergence acceleration was achieved by applying local time-stepping and a multigrid technique.

In this paper, the Lax-Friedrichs' type of dissipative flux in Jameson's scheme will be replaced by Roe's dissipative flux. It will be shown that this results in a scheme which captures shocks more accurately than Jameson's original scheme. However, the replacement of the dissipative flux term results in an increase of the calculation time.

In Section 2, the numerical schemes considered for solving the two-dimensional Euler equations are described. The treatment of the boundaries of the computational domain is discussed in Section 3. In Section 4, the Runge-Kutta time-stepping procedure is explained and in Section 5, several aspects of the multigrid technique are described. Finally, in Section 6, numerical results for inviscid flow around an aerofoil obtained using the new scheme are presented.

\section{THE NUMERICAL SCHEMES}

Fluid flow in two spatial dimensions can be modelled by a set of non-linear conservation laws, viz. conservation of mass, conservation of momentum and conservation of energy. The present 
investigation deals with the Euler equations, which follow from the Navier-Stokes equations when the viscous and heat conduction terms are ignored. In this paper, the Euler equations are used for simulating the inviscid flow around an aerodynamic body. For two spatial dimensions the time-dependent Euler equations can be written in the integral form

$$
\frac{\partial}{\partial t} \iint_{\Omega} \underline{q} \mathrm{~d} \Omega+\int_{\mathbf{\Omega} \Omega} \underline{\mathbf{f}}(\underline{q}) \cdot \mathbf{n} \mathrm{d} l=0
$$

where $t$ denotes the time. The state vector $\underline{q}$ is a four-dimensional vector, which contains the densities of the conserved quantities, i.e.

$$
q=\left[\begin{array}{c}
\rho \\
\rho u \\
\rho v \\
e
\end{array}\right]
$$

where $\rho, u, v$ and $e$ denote the density, the Cartesian velocity components and the total energy density, respectively.

The quantity $\Omega$ in equation (1) denotes an arbitrary control volume with boundary $\partial \Omega$ and outer unit normal $n$. The bold variables in equation (1) denote two-dimensional vectors in physical space. The product $\underline{\mathbf{f}} \cdot \mathbf{n}$ represents the flux that is directed out of the domain $\Omega$ through the boundaries. The flux tensor $\underline{\mathbf{f}}$ can be written as

$$
\underline{\mathbf{f}}(\underline{q})=\left[\begin{array}{ll}
\rho u \mathbf{i}_{x} & +\rho v \mathbf{i}_{y} \\
\left(\rho u^{2}+p\right) \mathbf{i}_{x}+\rho u v \mathbf{i}_{y} \\
\rho u v \mathbf{i}_{x} & +\left(\rho v^{2}+p\right) \mathbf{i}_{y} \\
\rho u H \mathbf{i}_{x} & +\rho v H \mathbf{i}_{y}
\end{array}\right],
$$

where $H=(e+p) / \rho$ is the specific enthalpy. The vectors $\mathbf{i}_{x}, \mathbf{i}_{y}$ form an orthogonal base of the two-dimensional space. For an ideal gas the pressure is defined as $p=(\gamma-1)\left(e-\frac{1}{2} \rho\left(u^{2}+v^{2}\right)\right)$, in which $\gamma$ denotes the specific heat ratio.

Numerical solution of the Euler equations requires the discretization of equation (1). The discretization of equation (1) follows the method of lines, i.e. discretization in space and time are done separately.

For the spatial discretization of equation (1) a finite volume method is used. To this end, a finite computational domain is defined by introducing far-field boundaries, which are sufficiently far away from the aerodynamic body. This computational domain is partitioned in quadrilateral cells with the help of a structured, boundary-conforming mesh. In the vertex-based approach chosen here the discretized variables are associated with the grid points $\underline{q}_{i, j}=\underline{q}\left(x_{i, j}, y_{i, j}\right)$, where $i=0, \ldots, m_{i}$ and $j=0, \ldots, m_{j}$. The integers $m_{i}$ and $m_{j}$ represent the dimensions of the grid in computational space. For each grid point a control volume is formed by connecting the midpoints of the four neighbouring cells with each other, as shown in Figure 1. It can be seen that the cell corresponding to grid point $\left(x_{i, j}, y_{i, j}\right)$ has four boundary segments or edges, which are numbered as $\left(i+\frac{1}{2}, j\right),\left(i-\frac{1}{2}, j\right),\left(i, j+\frac{1}{2}\right)$ and $\left(i, j-\frac{1}{2}\right)$. The surface area of the cell is denoted by $S_{i, j}$.

Taking the vertex-based control volume as $\Omega$, equation (1) has the form

$$
\frac{\mathrm{d} \underline{q}_{i, j}}{\mathrm{~d} t}+\frac{1}{S_{i, j}}\left[\underline{h}_{i+1 / 2, j}-\underline{h}_{i-1 / 2, j}+\underline{h}_{i, j+1 / 2}-\underline{h}_{i, j-1 / 2}\right]=0,
$$

where $\underline{h}_{i \pm 1 / 2, j}$ and $\underline{h}_{i, j \pm 1 / 2}$ represent the fluxes at the cell edges $\left(i \pm \frac{1}{2}, j\right)$ and $\left(i, j \pm \frac{1}{2}\right)$, respectively. The advantage of this formulation is that the discrete system remains conservative. 


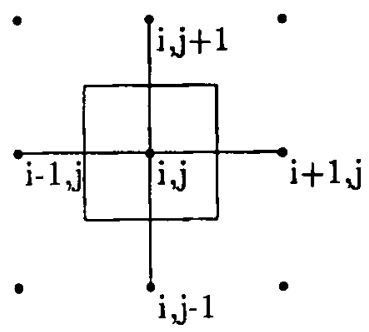

Figure 1. A two-dimensional cell in the vertex-based approach

In order to obtain a stable time-integration scheme for equation (4), the fluxes are split into two parts, namely a convective part and a dissipative (or diffusive) part. Thus,

$$
\underline{h}_{i+1 / 2, j}=\underline{C}_{i+1 / 2, j}-\underline{D}_{i+1 / 2, j}
$$

Hence, the semi-discrete relation (4) can be rewritten as

$$
\frac{\mathrm{d} \underline{q}_{i, j}}{\mathrm{~d} t}+\frac{1}{S_{i, j}}\left[\underline{C}_{i, j}-\underline{D}_{i, j}\right]=0,
$$

with the convective part

$$
\underline{C}_{i, j}=\underline{C}_{i+1 / 2, j}-\underline{C}_{i-1 / 2, j}+\underline{C}_{i, j+1 / 2}-\underline{C}_{i, j-1 / 2},
$$

and the dissipative part

$$
\underline{D}_{i, j}=\underline{D}_{i+1 / 2, j}-\underline{D}_{i-1 / 2, j}+\underline{D}_{i, j+1 / 2}-\underline{D}_{i, j-1 / 2} \text {. }
$$

The convective flux component through the edge $(i+1 / 2, j)$ is then calculated by taking an arithmetic average of the flux tensor

$$
\underline{C}_{i+1 / 2, j}=l_{i+1 / 2, j} \frac{\underline{\mathbf{f}}\left(\underline{q}_{i, j}\right)+\underline{\mathbf{f}}\left(\underline{q}_{i+1, j}\right)}{2} \cdot \mathbf{m}_{i+1 / 2, j},
$$

where $\mathbf{n}_{i+1 / 2, j}$ is the unit normal on the edge and $l_{i+1 / 2, j}$ is the length of the edge.

In the remainder of this section, the calculation of the dissipative part [equation (7)] will be discussed. The description of the dissipation models is done by defining the dissipative flux at the edge $(i+1 / 2, j)$ as a function of the variables $\underline{q}_{0, j}, \underline{q}_{1, j}, \ldots, \underline{q}_{m_{i}, j}, \mathbf{n}_{i+1 / 2, j}$ and $l_{i+1 / 2, j}$. In order to show how the shock-capturing of Jameson's scheme can be improved, Jameson's dissipation model will first be discussed below. It appears that a Lax-Friedrichs' type of dissipative flux is responsible for the shock-capturing in Jameson's scheme. The Lax-Friedrichs' dissipation model will then be described. The proposed new scheme is constructed from Jameson's scheme by replacing Lax-Friedrichs' dissipation model with Roe's dissipation model. In order to introduce this, Roe's dissipation model will subsequently be discussed. Finally, at the end of this section the new scheme will be presented.

\subsection{Jameson's dissipation model}

The dissipation model considered here initially was proposed by Jameson. ${ }^{2}$ The resulting scheme [equation (5)] is pseudo-second-order accurate in space. The idea behind Jameson's scheme is to use a fourth-order difference term in the calculation of the flux differences in equation 
(7). This provides a base level of dissipation sufficient to prevent odd-even decoupling throughout the domain, but not sufficient to prevent oscillations in the numerical solution in the neighbourhood of shock waves. In order to capture a shock wave, an additional second-order difference term is added locally in response to a sensor designed to detect discontinuities in the pressure. The dissipative flux can be written as

$$
\underline{D}_{i+1 / 2, j}=l_{i+1 / 2, j}\left[\varepsilon_{i+1 / 2, j}^{(2)} \lambda_{i+1 / 2, j} \Delta_{i+1 / 2, j} \underline{q}-\varepsilon_{i+1 / 2, j}^{(4)} \lambda_{i+1 / 2, j} \Delta_{i+1 / 2, j}^{3} \underline{q}\right]
$$

with $\Delta_{i+1 / 2, j}^{3} \underline{q}=q_{i+2, j}-3 \underline{q}_{i+1, j}+3 \underline{q}_{i, j}-\underline{q}_{i-1, j}$ and $\lambda_{i+1 / 2, j}=\lambda\left(\underline{q}_{i+1 / 2, j}, \mathbf{n}_{i+1 / 2, j}\right)$ the maximum absolute eigenvalue of the flux Jacobian matrix. The flux Jacobian matrix is defined as

$$
A(\underline{q}, \mathbf{n})=\frac{\mathrm{d} \underline{\mathbf{f}}}{\mathrm{d} q}(\underline{q}) \cdot \mathbf{n}
$$

The maximum absolute eigenvalue of the flux Jacobian matrix is

$$
\lambda(\underline{q}, \mathbf{n})=\left|u_{\mathbf{n}}\right|+c,
$$

with $u_{\mathrm{n}}$ the velocity normal to the edge and $c$ the sound speed defined as $c^{2}=\gamma p / \rho$. The normal velocity and the sound speed in equation (11) on the edge $(i+1 / 2, j)$ then follow from

$$
\begin{aligned}
u_{\mathrm{n}} & =n_{1} \frac{u_{1}+u_{2}}{2}+n_{2} \frac{v_{1}+v_{2}}{2}, \\
c & =\frac{c_{1}+c_{2}}{2},
\end{aligned}
$$

in which the subscripts 1 and 2 refer to the grid points $(i, j)$ and $(i+1, j)$, respectively.

The coefficients $\varepsilon^{(2)}$ and $\varepsilon^{(4)}$ in equation (9) are functions of the value of the pressure discontinuity sensor $v_{i+1 / 2, j}$ given by

$$
v_{i+1 / 2, j}=\max \left\{v_{i+1, j}, v_{i, j}\right\},
$$

where

$$
v_{i, j}=\frac{\left|p_{i+1, j}-2 p_{i, j}+p_{i-1, j}\right|}{p_{i+1, j}+2 p_{i, j}+p_{i-1, j}} .
$$

Thus, the shock sensor is $O\left(h^{2}\right)$ in a smooth field and $O(1)$ near a shock. Jameson writes the adaptive coefficients as

$$
\begin{aligned}
& \varepsilon_{i+1 / 2, j}^{(2)}=\min \left(1 / 2, \kappa^{(2)} v_{i+1 / 2, j}\right), \\
& \varepsilon_{+1 / 2, j}^{(4)}=\max \left(0, \kappa^{(4)}-\alpha^{(4)} v_{i+1 / 2, j}\right),
\end{aligned}
$$

where $\kappa^{(2)}, \kappa^{(4)}$ and $\alpha^{(4)}$ are constants.

The difference formula $\Delta_{i+1 / 2, j}^{3} \underline{q}$ is undefined at the boundaries of the computational domain. There it is replaced by

and

$$
\Delta_{1 / 2, j}^{3} \underline{q}=\underline{q}_{2, j}-2 \underline{q}_{1, j}+\underline{q}_{0, j}
$$

$$
\Delta_{m_{1}-1 / 2, j}^{3} \underline{q}=-\underline{q}_{m_{i}, j}+2 \underline{q}_{m_{i}-1, j}-\underline{q}_{m_{i}-2, j},
$$

appropriately. Also the shock sensor [equation (13)] is set equal to zero:

$$
v_{-1, j}=v_{0, j}=v_{m_{i, j}}=v_{m_{i}+1, j}=0 \text {. }
$$


In order to show how the shock-capturing facility of Jameson's scheme can be improved, the dissipative term which is responsible for the shock-capturng will be examined more carefully.

\subsection{Lax-Friedrichs' dissipation model}

The Lax-Friedrichs' scheme described in Reference 3 is a central difference scheme, which is first-order accurate in space. It is well-known that steady-state solutions obtained with this scheme are smooth solutions. Hence, the Lax-Friedrichs' dissipative flux is generally too dissipative. It is given by

$$
\underline{D}_{i+1 / 2, j}=\frac{1}{2} l_{i+1 / 2, j} \lambda_{i+1 / 2, j} \Delta_{i+1 / 2, j} \underline{q},
$$

where $\lambda_{i+1 / 2, j}$ is calculated according to equation (11). Considering that Jameson's scheme contains the Lax-Friedrichs' type of dissipative flux premultiplied with the adaptive coefficient $\varepsilon^{(2)}$, the idea arises to replace Lax-Friedrichs' dissipation model by another, less dissipative, alternative. In the following section one choice will be examined in more detail.

\subsection{Roe's dissipation model}

Roe's scheme is a first-order-accurate upwind scheme, which belongs to the class of fluxdifference splitting schemes. This means, in fact, that the flux differences in both directions in equation (4) are calculated by using first-order-accurate forward or backward differences, depending upon the sign of the eigenvalue of the flux Jacobian matrix. The method is described in Reference 1 . The dissipative flux becomes

where

$$
\underline{D}_{i+1 / 2, j}=\frac{1}{2} l_{i+1 / 2, j}\left|A_{i+1 / 2, j}\right| \Delta_{i+1 / 2, j} \underline{q},
$$

$$
\Delta_{i+1 / 2, j} \underline{q}=\underline{q}_{i+1, j}-\underline{q}_{i, j} \text { and } A_{i+1 / 2, j}=\tilde{A}\left(\underline{q}_{i, j}, \underline{q}_{i+1, j}, \mathbf{n}_{i+1 / 2, j}\right)
$$

is an approximation of the flux Jacobian matrix [equation (10)]. Here $\underline{q}_{i+1 / 2, j}$ is referred to as Roe's average state. It can easily be seen that Roe's dissipative flux [equation (17)] is generally less dissipative than Lax-Friedrichs' dissipative flux [equation (16)]. It is well-known that the flux Jacobian matrix of the Euler equations (10) in one direction has a complete set of eigenvectors, so that the flux Jacobian matrix can be diagonalized as $A(q, \mathbf{n})=R(q, \mathbf{n}) \Lambda(q, \mathbf{n}) L(q, \mathbf{n})$, where $R$ denotes the right eigenvector matrix, $L$ is the left eigenvector matrix and $\Lambda$ is the eigenvalue matrix. The absolute value of the flux Jacobian matrix in equation (17) is defined as $|A|=R|\Lambda| L$.

The diagonal matrix $\Lambda(\underline{q}, \mathbf{n})$ contains the eigenvalues of the flux Jacobian matrix [equation (10)]:

$$
\Lambda(\underline{q}, \mathbf{n})=\left[\begin{array}{cccc}
u_{\mathrm{n}} & 0 & 0 & 0 \\
0 & u_{\mathrm{n}} & 0 & 0 \\
0 & 0 & u_{\mathrm{n}}+c & 0 \\
0 & 0 & 0 & u_{\mathrm{n}}-c
\end{array}\right],
$$

where $u_{\mathrm{n}}=n_{1} u+n_{2} v$ is the component of the velocity normal to the edge and $c$ is the sound speed. The right eigenvector matrix is then

$$
R(\underline{q}, \mathbf{n})=\left[\begin{array}{cccc}
1 & 0 & \beta & \beta \\
u & n_{2} & \beta\left(u+n_{1} c\right) & \beta\left(u-n_{1} c\right) \\
v & -n_{1} & \beta\left(v+n_{2} c\right) & \beta\left(v-n_{2} c\right) \\
\phi^{2} /(\gamma-1) & n_{2} u-n_{1} v & \beta\left(H+u_{\mathrm{n}} c\right) & \beta\left(H-u_{\mathrm{n}} c\right)
\end{array}\right]
$$


and the left eigenvector matrix is

$$
L(\underline{q}, \mathbf{n})=\left[\begin{array}{cccc}
1-\phi^{2} / c^{2} & (\gamma-1) u / c^{2} & (\gamma-1) v / c^{2} & -(\gamma-1) / c^{2} \\
-n_{2} u+n_{1} v & n_{2} & -n_{1} & 0 \\
\phi^{2}-u_{\mathrm{n}} c & n_{1} c-(\gamma-1) u & n_{2} c-(\gamma-1) v & \gamma-1 \\
\phi^{2}+u_{\mathrm{n}} c & -n_{1} c-(\gamma-1) u & -n_{2} c-(\gamma-1) v & \gamma-1
\end{array}\right],
$$

where $\beta=1 / 2 c^{2}$ and $\phi^{2}=\frac{1}{2}(\gamma-1)\left(u^{2}+v^{2}\right)$.

In order to determine Roe's average state vector needed in equation (18), Roe locally linearizes the flux Jacobian matrix. Consider the left state $\underline{q}_{1}$ and the right state $\underline{q}_{\mathrm{r}}$ and denote by $q_{1, \mathrm{r}}$ Roe's average state. The locally linearized flux Jacobian matrix $\tilde{A}$ then has to satisfy the following four conditions:

1. The matrix constitutes a linear mapping from the vector space $q$ to the vector space $\underline{\mathbf{f}}$.

2. $\tilde{A}(\underline{q}, \underline{q}, \mathbf{n})=A(\underline{q}, \mathbf{n})$.

3. $\left(\underline{\mathbf{f}}_{1}-\underline{\mathbf{f}}_{\mathrm{r}}\right) \cdot \mathbf{n}=\tilde{A}\left(\underline{q}_{1}, \underline{q}_{\mathrm{r}}, \mathbf{n}\right) \times\left(\underline{q}_{1}-\underline{q}_{\mathrm{r}}\right)$.

4. The eigenvectors of $A$ are linearly independent.

Here $\mathbf{f}$ is the flux tensor given by equation (3). The second condition above is needed for consistency. The third condition above makes the scheme conservative. The third and fourth conditions are necessary and sufficient for the algorithm to recognize shock waves.

Roe's average state vector $\underline{q}_{1, \mathrm{r}}$ is derived such that $A\left(\underline{q}_{1, \mathrm{r}}, \mathbf{n}\right)=\tilde{A}\left(\underline{q}_{1}, \underline{q}_{\mathrm{r}}, \mathbf{n}\right)$ holds. The explicit form of Roe's average state vector is given by

$$
\begin{aligned}
& \rho_{1, \mathrm{r}}=\frac{\rho_{1}+d \rho_{\mathrm{r}}}{1+d}, \\
& u_{1, \mathrm{r}}=\frac{u_{1}+d u_{\mathrm{r}}}{1+d}, \\
& v_{1, \mathrm{r}}=\frac{v_{1}+d v_{\mathrm{r}}}{1+d}, \\
& h_{1, \mathrm{r}}=\frac{h_{1}+d h_{\mathrm{r}}}{1+d},
\end{aligned}
$$

in which $d=\sqrt{\rho_{\mathrm{r}}} / \sqrt{\rho_{\mathrm{l}}}$. The speed of sound follows from $c_{1, \mathrm{r}}^{2}=(\gamma-1)\left(h_{1, \mathrm{r}}-\frac{1}{2}\left(u_{1, \mathrm{r}}^{2}+v_{1, \mathrm{r}}^{2}\right)\right)$.

An additional requirement for Roe's dissipation model is necessary, as shown in Reference 4 if at least one of the eigenvalues of the flux Jacobian matrix [equation (10)] is zero. In this case the scheme would lead to a decrease of the entropy, which is impossible according to the second law of thermodynamics. In order to prevent entropy violation, a mechanism called the entropy fix is used. To this end, the eigenvalues are scaled with a specified portion $\delta$ of the maximum absolute eigenvalue $\lambda_{\max }$, i.e.

$$
|\lambda|=\max \left\{|\lambda|, \delta \lambda_{\max }\right\},
$$

where $\lambda$ denotes an eigenvalue of the Jacobian matrix [equation (10)]. Note that the choice $\delta=1$ in equation (21) turns Roe's dissipation model into Lax-Friedrichs' dissipation model.

\subsection{The new dissipation model}

The new dissipation model discussed here is based on both Roe's and Jameson's dissipation models. The shock-capturing of Jameson's scheme is improved by replacing the Lax-Friedrichs' 
dissipative flux [equation (16)] present in Jameson's scheme by Roe's dissipative flux [equation (17)]. As a matter of fact, any other first-order-accurate flux difference scheme such as the polynomial flux difference splitter of Dick ${ }^{5}$ or Osher's flux difference splitter ${ }^{6}$ could be considered for replacement of the Lax-Friedrichs' type of dissipation.

The replacement of Lax-Friedrichs' dissipative flux by Roe's dissipative flux results in a hybrid scheme, which takes the characteristic directions more into account. So, the proposed new scheme will be more accurate near shocks than Jameson's scheme. The proposed dissipative flux is

$$
\underline{D}_{i+1 / 2, j}=l_{i+1 / 2, j}\left[\varepsilon_{i+1 / 2, j}^{(2)}\left|A_{i+1 / 2, j}\right| \Delta_{i+1 / 2, j} \underline{q}-\varepsilon_{i+1 / 2, j}^{(4)} \lambda_{i+1 / 2, j} \Delta_{i+1 / 2, j}^{3} q\right],
$$

where the same notation is used as before. The elements of the flux Jacobian matrix $A_{i+1 / 2, j}$ and the maximum absolute eigenvalue $\lambda_{i+1 / 2, j}$ are calculated with Roe's average state vector [equation (20)]. Due to the fact that Roe's dissipation model is used, the entropy fix is necessary.

The shock sensor given by equation (13) is replaced by

$$
v_{i+1 / 2, j}=\max \left\{v_{i+2, j}, v_{i+1, j}, v_{i, j}, v_{i-1, j}\right\} .
$$

The calculation of the adaptive coefficients $\varepsilon^{(2)}, \varepsilon^{(4)}$ and the treatment of the difference formula $\Delta_{i+1 / 2, j}^{3} \underline{q}$ and the pressure sensor at the boundaries of the computational domain remain the same as in Jameson's scheme.

\section{BOUNDARY CONDITIONS}

In the numerical experiments for inviscid flow around an aerofoil described in Section 6, an O-type co-ordinate mesh will be used in order to obtain the steady-state solution of the discretized Euler equations. Due to the use of a mesh, boundary conditions become necessary. In this section two types of boundary conditions are discussed, firstly a far-field boundary condition and secondly a solid-wall boundary condition. The first boundary condition applied at the far-field boundary exists due to finiteness of the domain. A circulation correction is used in order to diminish the amount of computational work. The second condition is used at the aerofoil. Special treatment will be necessary at the trailing edge of the aerofoil.

At a far-field boundary, subsonic inflow or outflow is permitted. Depending upon whether the far-field boundary is an inflow or an outflow boundary, the Riemann invariants are prescribed or extrapolated. The locally one-dimensional Riemann invariants are as shown in Table I. Here $s=\ln \left(p / \rho^{\gamma}\right)$ denotes the entropy and $u_{t}$ the tangential velocity component.

If the eigenvalue $\lambda>0$, the corresponding Riemann invariants are extrapolated linearly from their interior values. If $\lambda<0$, the corresponding Riemann invariant is set equal to its free-stream

Table I. The eigenvalues of the Jacobian matrix and their corresponding Riemann invariants

\begin{tabular}{lc}
\hline Eigenvalue $\lambda$ & Riemann invariants \\
\hline$u_{\mathrm{n}}$ & $s, u_{\mathrm{t}}$ \\
$u_{\mathrm{n}}+c$ & $u_{\mathrm{n}}+2 \frac{c}{\gamma-1}$ \\
$u_{\mathrm{n}}-c$ & $u_{\mathrm{n}}-2 \frac{c}{\gamma-1}$ \\
\hline
\end{tabular}


value. Once all four Riemann invariants are available at a far-field edge, the four components of the state vector $q$ there can be calculated.

If the free-stream values are used for computing the state vectors at the far-field boundary, the size of the domain must be large, viz. the outer boundary must lie at 100 chord lengths of the aerofoil. The consequence of this is that the amount of computational work is large, because a large number of grid points becomes necessary. In order to diminish the amount of computational work, the extent of the domain can be reduced by making a circulation correction to the free-stream values. This is done by imposing a compressible potential vortex solution, which is added as a perturbation to the free-stream values $\left(u_{\infty}=q_{\infty} \cos \alpha\right.$ and $\left.v_{\infty}=q_{\infty} \sin \alpha\right)$, where $q_{\infty}$ denotes the free-stream velocity and $\alpha$ is the angle of attack of the incoming flow. The perturbed far-field boundary velocities are then calculated as

and

$$
u_{\mathrm{f}}=u_{\infty}+\frac{\xi \Gamma \sin \theta}{2 \pi r\left[1-M_{\infty}^{2} \sin ^{2}(\theta-\alpha)\right]}
$$

$$
v_{\mathrm{f}}=v_{\infty}-\frac{\xi \Gamma \cos \theta}{2 \pi r\left[1-M_{\infty}^{2} \sin ^{2}(\theta-\alpha)\right]},
$$

where the circulation $\Gamma=\frac{1}{2} q_{\infty} L c_{1}, L$ is the chord length of the aerofoil, $c_{1}$ is the lift coefficient at the surface of the aerofoil, $M_{\infty}$ is the free-stream Mach number, $\xi=\sqrt{ }\left(1-M_{\infty}^{2}\right)$. This choice for $\xi$ implies that only subsonic free-stream Mach numbers are allowed. Furthermore, $r$ and $\theta$ are the polar co-ordinates of a grid point on the outer boundary relative to an origin at the quarter point on the aerofoil centre line. Requiring that the free-stream enthalpy is constant at the outer boundary results in the corrected speed of sound,

$$
c_{\mathrm{f}}^{2}=(\gamma-1)\left[H_{\infty}-\frac{1}{2}\left(u_{\mathrm{f}}^{2}+v_{\mathrm{f}}^{2}\right)\right] .
$$

The quantities $u_{\mathrm{f}}, v_{\mathrm{f}}$ and $c_{\mathrm{f}}$ are used instead of the free-stream values at the far-field boundary.

The boundary procedure at the solid wall can be explained as follows. In case of the Euler equations there is only one physical boundary condition at the solid wall, namely $u_{n}=0$, where $u_{n}$ is the component of the velocity normal to the wall. In order to determine the state vector with the conserved quantities at the solid wall, firstly the density, the velocity components and the pressure are linearly extrapolated in the normal direction. Secondly, the normal velocity at the solid wall is set equal to zero. The energy density then follows from the pressure, density and the velocity components.

Since in an O-type mesh (as used in Section 6) no grid points normal to the solid wall are available at the trailing edge, there the extrapolation is performed using the state vectors on the wall in the upstream direction. The result of this is that the trailing edge becomes a bivalent point, which makes it possible to capture a contact discontinuity.

\section{RUNGE-KUTTA TIME-STEPPING WITH FROZEN DISSIPATION}

In order to compute an approximate steady-state solution of the system which consists of equation (5) for each interior grid point and the boundary conditions described in the previous section, explicit Runge-Kutta time-stepping with frozen dissipation can be applied if a large number of time-steps is performed. Equation (5) can be written more generally as

$$
S \frac{\mathrm{d} q}{\mathrm{~d} t}+N(\underline{q})=\underline{g}
$$


where $S$ denotes the surface area and $g$ is a possible right-hand-side vector, which equals zero for equation (5). The operator $N$ is

$$
N(\underline{q})=C(\underline{q})-D(\underline{q}) \text {. }
$$

In order to explain the Runge-Kutta procedure applied to equation (27), the defect vector $\underline{d}$ is introduced as

$$
\underline{d}=N(\underline{q})-\underline{g} .
$$

Note that the surface area $S$ is not incorporated in the calculation of this defect vector.

The advancement of a time-step $\Delta t$ with a time-explicit $k$-stage Runge-Kutta scheme can now be written as

$$
\begin{aligned}
\underline{q}^{(0)} & =\underline{q}(t) \\
\underline{q}^{(1)} & =\underline{q}^{(0)}-\alpha_{1} S^{-1} \Delta t \underline{d}^{(0)}, \\
\underline{q}^{(2)} & =\underline{q}^{(0)}-\alpha_{2} S^{-1} \Delta t \underline{d}^{(1)}, \\
& \cdots \\
\underline{q}^{(k-1)} & =\underline{q}^{(0)}-\alpha_{k-1} S^{-1} \Delta t \underline{d}^{(k-2)}, \\
\underline{q}^{(k)} & =\underline{q}^{(0)}-\alpha_{k} S^{-1} \Delta t \underline{d}^{(k-1)}, \\
\underline{q}(t+\Delta t) & =\underline{q}^{(k)},
\end{aligned}
$$

where $\alpha_{i}$ are constants, with $\alpha_{k}=1$ for consistency. The coefficients $\alpha_{i}$ and the number of Runge-Kutta stages $k$ are not fixed, which makes the Runge-Kutta routine a flexible time-step procedure. Convergence acceleration can be achieved by taking a local time-step in each cell of the computational domain.

These local time-steps must satisfy a Courant-Friedrichs-Lewy (CFL) condition ${ }^{7}$ in order for the time integration [equation (30)] to be stable. The local time-step for the cell $(i, j)$ can be calculated as in Reference 8. There it is shown that if the CFL number has the same value in each direction, one-dimensional stability is sufficient for two-dimensional stability. The local time-step is then equal to

$$
\Delta t_{i, j}=\frac{1}{1 / \Delta t_{i}+1 / \Delta t_{j}},
$$

where $\Delta t_{i}$ and $\Delta t_{j}$ denote the maximum time-steps allowed in the $i$ - and $j$-direction, respectively, i.e.

$$
\begin{aligned}
\Delta t_{i} & =\frac{\sigma S_{i, j}}{\max \left\{l_{i+1 / 2, j} \lambda_{i+1 / 2, j}, l_{i-1 / 2, j} \lambda_{i-1 / 2, j}\right\}}, \\
\Delta t_{j} & =\frac{\sigma S_{i, j}}{\max \left\{l_{i, j+1 / 2} \lambda_{i, j+1 / 2}, l_{i, j-1 / 2} \lambda_{i, j-1 / 2}\right\}},
\end{aligned}
$$

where $\sigma$ denotes the CFL number.

In order to reduce the calculation time, Jameson ${ }^{2}$ freezes the dissipative fluxes [equation (7)] present in the defect vector during the Runge-Kutta stages. As a consequence, the defect vector in the $k$ th Runge-Kutta stage is calculated as (see also Radespiel $^{9}$ )

$$
\underline{d}^{(k)}=C\left(\underline{q}^{(k)}\right)-\sum_{r=0}^{k} \gamma_{i r} D\left(\underline{q}^{(r)}\right)-\underline{g}
$$


where the weighing factors $\gamma_{k r}$ are subject to the constraint

$$
\sum_{r=0}^{k} \gamma_{k r}=1, \quad k \geq 0 \text {. }
$$

\section{MULTIGRID}

The application of the Runge-Kutta technique to a single-grid calculation with a small mesh size requires a very large number of Runge-Kutta time-steps. This is due to the fact that Runge-Kutta time-stepping is unable to damp Fourier modes with a long wavelength quickly. In a multigrid technique these modes can be damped by using coarser grids.

The multigrid technique used in the present work is described in Reference 10 and consists of two parts. In the first part, a full multigrid procedure (FMG) is employed to compute an accurate initial solution vector for the finest grid. In the second part, the full approximation scheme (FAS) is used to compute the steady-state solution at machine accuracy. For this purpose, a previously set number of $\mathrm{V}$-cycles is performed such that the solution converges.

In the restriction of the state vector $q$ on the fine grid (denoted by 1 ) to the coarse grid (denoted by $L$ ), the injection operator

$$
\underline{q}_{i, j}^{\mathrm{L}}=\underline{q}_{2 i, 2 j}^{\mathrm{l}},
$$

for $i=0, \ldots, m_{i}$ and $j=0, \ldots, m_{j}$ is used. For the restriction of the defect vector two types of operators were used. This is due to the fact that at boundaries of the computational domain the following definition of the defect vector will be used:

$$
\underline{d}=\underline{q}(t)-\underline{q}(t+\Delta t)-\underline{g},
$$

where $q(t)$ denotes the state vector at time $t$ and $\underline{g}$ the right-hand-side vector. The full weighting operator used to restrict the defect vector is

$$
\begin{aligned}
\underline{d}_{i, j}^{\mathrm{L}}= & \frac{1}{4}\left[\underline{d}_{2 i+1,2 j+1}^{1}+2 \underline{d}_{2 i+1,2 j}^{1}+\underline{d}_{2 i+1,2 j-1}^{1}+2 \underline{d}_{2 i, 2 j+1}^{1}+4 \underline{d}_{2 i, 2 j}^{1}+2 \underline{d}_{2 i, 2 j-1}^{1}\right. \\
& \left.+\underline{d}_{2 i-1,2 j+1}^{1}+2 \underline{d}_{2 i-1,2 j}^{1}+\underline{d}_{2 i-1,2 j-1}^{1}\right],
\end{aligned}
$$

for $i=1, \ldots, m_{i}-1$ and $j=1, \ldots, m_{j}-1$. This operator has been premultiplied with a factor 4 , because in the calculation of the defect vector [equation (29)] the control volume has not been accounted for. At the boundary of the computational domain, the injection operator used for the restriction of the defect vector is

$$
\underline{d}_{i, j}^{L}=4 \underline{d}_{2 i, 2 j}^{1} \text {. }
$$

In the prolongation the corrections to the state vectors on the coarse grid are interpolated bilinearly to obtain the state vectors on the next finer grid.

\section{NUMERICAL RESULTS}

In this section, the numerical results for inviscid flow around an aerofoil for the schemes described in Section 2 will be discussed. The numerical experiments are performed with Jameson's, Roe's and the proposed new scheme. The Lax-Friedrichs' scheme will not be used in the experiments, because it is generally too dissipative for these applications.

A multigrid technique and Runge-Kutta time-stepping have been applied in order to compute a steady-state solution of the discretized Euler equations. Firstly, the two-dimensional subsonic 
flow around the NACA0012 aerofoil at free-stream Mach number $M_{\infty}=0.63$ and angle of attack $\alpha=2^{\circ}$ has been simulated in order to demonstrate that Jameson's dissipation model and the new dissipation model result in the same steady-state solution for a shock-free flow. Secondly, a test case with a strong shock where the free-stream Mach number $M_{\infty}=0.8$ and the angle of attack $\alpha=1.25^{\circ}$ has been taken. It will be shown that the new scheme resolves the strong shock more accurately than Jameson's scheme. In addition, a comparison between Jameson's scheme and the new scheme with respect to convergence rate and calculation time needed to reach a steady-state solution has been made. Finally, a grid refinement study for the new scheme has been carried out. In this study, the convergence behaviour of the lift and drag coefficient for grid refinement were examined. An O-type mesh containing $257 \times 129$ grid points (see Figure 2) was used for the first two test cases. This implies that 257 grid points were distributed over the aerofoil surface, whereas 129 grid points were used in the normal direction to this surface. The far-field boundary lies approximately at 20 chord lengths away from the aerofoil.

The system of ordinary differential equations (4) was advanced in time with a five-stage Runge-Kutta time-stepping scheme for each dissipation model. A hybrid scheme in which the dissipative terms were evaluated at the first, third and fifth stages of the scheme was used. The stage coefficients were taken as

$$
\alpha_{1}=1 / 4, \quad \alpha_{2}=1 / 6, \quad \alpha_{3}=3 / 8, \quad \alpha_{4}=1 / 2, \quad \alpha_{5}=1 .
$$

The weighing factors in the Runge-Kutta procedure [equation (33)] were set to ${ }^{9}$

$$
\begin{aligned}
& \gamma_{00}=1 \\
& \gamma_{10}=1, \quad \gamma_{11}=0, \\
& \gamma_{20}=1-\overline{\gamma_{3}}, \quad \gamma_{21}=0, \quad \gamma_{22}=\overline{\gamma_{3}}, \\
& \gamma_{301}=1-\overline{\gamma_{3}}, \quad \gamma_{31}=0, \quad \gamma_{32}=\overline{\gamma_{3}}, \quad \gamma_{33}=0, \\
& \gamma_{40}=\left(1-\overline{\gamma_{3}}\right)\left(1-\overline{\gamma_{5}}\right), \quad \gamma_{41}=0, \quad \gamma_{42}=\overline{\gamma_{3}}\left(1-\overline{\gamma_{5}}\right), \quad \gamma_{43}=0, \quad \gamma_{44}=\overline{\gamma_{5}},
\end{aligned}
$$

where $\overline{\gamma_{3}}=0.56$ and $\overline{\gamma_{5}}=0.44$. $^{11}$

In the multigrid technique five grid levels have been used. So, the coarsest mesh contained $17 \times 9$ grid points. In the full multigrid stage, three V-cycles were applied at every grid level until the finest mesh has been reached. Subsequently, $100 \mathrm{~V}$-cycles were employed in order to obtain the steady-state solution. In the presentation of the numerical results the following quantities will be shown:

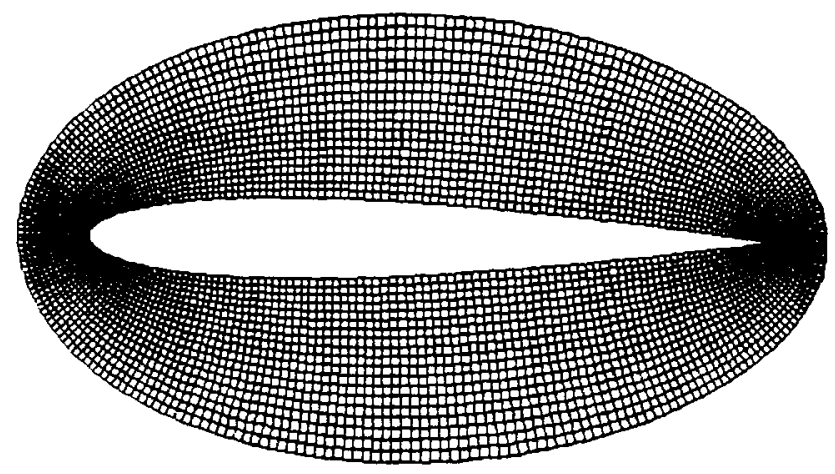

Figure 2. An enlarged view of the O-type mesh 
the pressure coefficient

$$
c_{p}=\frac{p-p_{\infty}}{\frac{1}{2} \rho_{\infty} U_{\infty}^{2}}
$$

the total pressure

$$
p_{1}=p\left[1+\frac{1}{2}(\gamma-1) M^{2}\right]^{\gamma /(\gamma-1)}
$$

the lift coefficient

$$
c_{1}=\frac{1}{\frac{1}{2} \rho_{\infty} U_{\infty}^{2} c_{\infty}} \oint-p\left[-n_{1} \sin \alpha+n_{2} \cos \alpha\right] \mathrm{d} s,
$$

the drag coefficient

$$
c_{\mathrm{d}}=\frac{1}{\frac{1}{2} \rho_{\infty} U_{\infty}^{2} c_{\infty}} \oint-p\left[n_{1} \cos \alpha+n_{2} \sin \alpha\right] \mathrm{d} s .
$$

Here $\rho_{\infty}, U_{\infty}=\sqrt{ }\left(u_{\infty}^{2}+v_{\infty}^{2}\right), p_{\infty}$ and $c_{\infty}$ denote the free-stream values of density, velocity, pressure and speed of sound, $M=u / c$ is the Mach number and $n=\left[n_{1}, n_{2}\right]$ is the outward unit normal to the solid wall. The trapezoidal rule was applied to evaluate the integrals for the lift and the drag coefficients. To measure the convergence rate, the discrete $L_{2}$ norm of the first component of the defect vector [equation (29)] was taken, i.e. the norm

$$
\left.\left\|d_{1}\right\|_{2}=\sqrt{\left(\sum_{i=1}^{m_{i}-1} \sum_{j=2}^{m_{j}-1} d_{i, j, 1}^{2} / S_{i, j}^{2}\right.}\right),
$$

where $S_{i, j}$ denotes the control volume and the summation is taken over the interior grid points of the O-type grid.

\subsection{The first test case with $M_{\infty}=0.63$ and $\alpha=2^{\circ}$}

This is a standard test case which has received wide attention in the literature. ${ }^{12-15}$ At subsonic free-stream conditions a shock-free flow field develops. Theoretically, the drag coefficient equals zero and the total pressure is constant. However, due to discretization errors the drag coefficient will be non-zero.

For this subsonic test case, the parameter $\kappa^{(2)}$ can be set equal to zero due to the fact that there are no shocks present in the steady-state solution. For this choice of $\kappa^{(2)}$, Jameson's scheme and the new scheme are identical. However, in the numerical experiments the multigrid procedure followed appeared to be unstable. Therefore, a small amount of second-order artificial dissipation was added, i.e. $\kappa^{(2)}>0$.

The parameter settings for Roe's, Jameson's and the new dissipation model were as shown in Table II. The parameters $\kappa^{(2)}, \kappa^{(4)}, \alpha^{(4)}$ and $\delta$ were chosen such that the steady-state solution of the transonic test case was oscillation-free. Therefore, the dissipative coefficient $\kappa^{(2)}$ in Jameson's scheme was set equal to $1 \cdot 5$, whereas for the new scheme it was set equal to $10 \cdot 0$. The motivation for choosing a larger $\kappa^{(2)}$ in the new scheme is that the Lax-Friedrichs' dissipation model (in Jameson's scheme) is more dissipative than Roe's dissipation model (in the new scheme). The parameter $\delta$ in Roe's dissipative flux, which is needed to prevent entropy violation, was set equal to 0.02 . In the transonic test case it appeared that $\delta$ had to be non-zero in order to obtain a stable multigrid routine.

The CFL number $\sigma$ was chosen in such a way that optimal damping in the multigrid procedure was achieved. For Roe's scheme $\sigma=3 \cdot 0$, for Jameson's scheme $\sigma=3.2$ and for the new scheme 
Table II. The parameter settings for the subsonic test case $M_{\infty}=0.63$ and $\alpha=2^{\circ}$ and for the transonic test case $M_{\infty}=0.8$ and $\alpha=1.25^{\circ}$

\begin{tabular}{lcccccccc}
\hline Dissipation model & $\sigma$ & $\mu_{0}$ & $\mu_{1}$ & $\mu_{2}$ & $\kappa^{(2)}$ & $\kappa^{(4)}$ & $\alpha^{(4)}$ & $\delta$ \\
\hline Roe & 3.0 & 10 & 4 & 4 & $-\overline{1}$ & $-\overline{-}$ & $-\overline{2.0}$ & 0.02 \\
Jameson & $3 \cdot 2$ & 10 & 5 & 5 & 1.5 & 0.03125 & - \\
New & $4 \cdot 0$ & 10 & 5 & 5 & $10 \cdot 0$ & 0.03125 & 3.0 & 0.02 \\
\hline
\end{tabular}

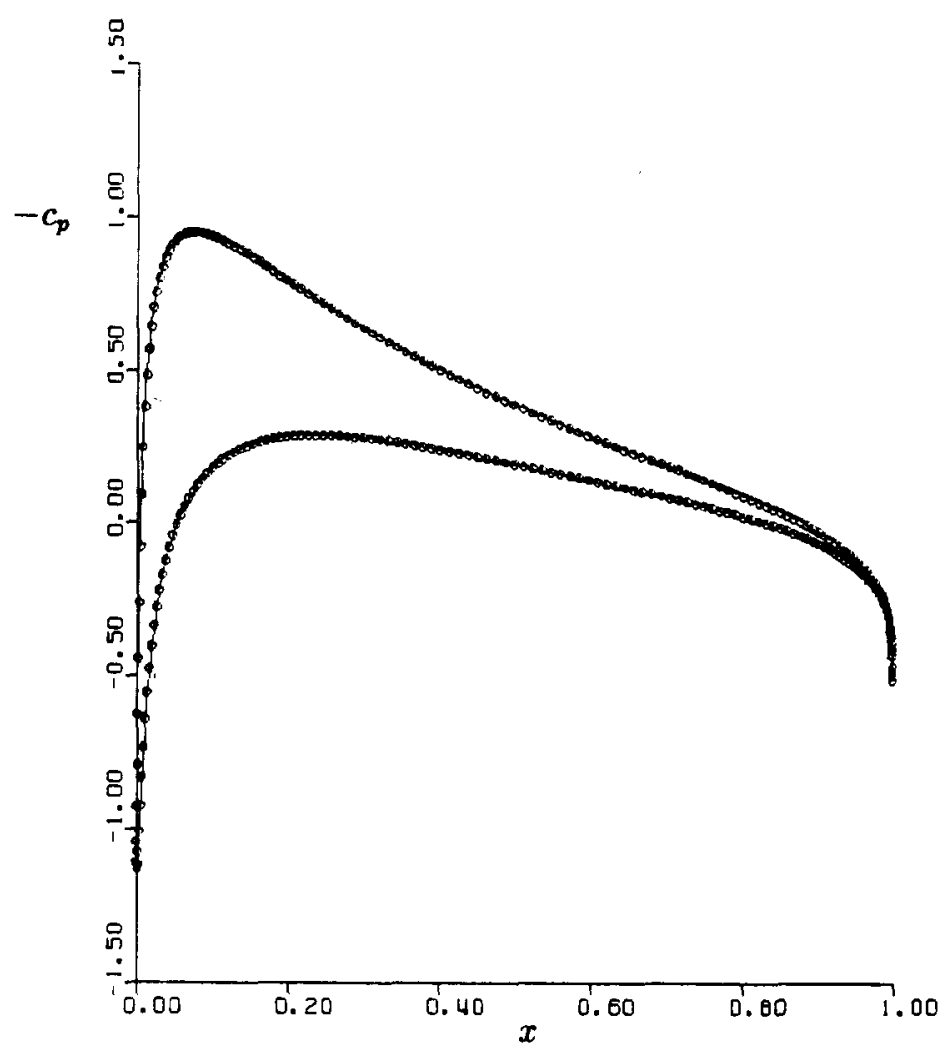

Figure 3. The pressure coefficient $c_{\mathrm{p}}$ along the surface $x$ of the aerofoil for $M_{\infty}=0.63$ and $\alpha=2^{\circ}$ for Roe's dissipation model on the $257 \times 129$ grid. In this picture and in all other pressure distributions the pressure coefficient and the chord length of the aerofoil are made dimensionless. Furthermore, in every pressure distribution the upper and the lower curves represent the pressure distributions at the upper and the lower surfaces of the aerofoil, respectively

$\sigma=4.0$ were used. In the numerical experiments it appeared that Jameson's and the new scheme need one additional pre-relaxation and post-relaxation compared to Roe's scheme. For all three schemes the steady-state solution was computed within machine accuracy. The pressure distributions along the aerofoil surface for Roe's, Jameson's and the new dissipation model are shown in Figures 3-5. One can see that Jameson's scheme and the new scheme result in identical pressure distributions. The pressure distribution obtained with Roe's scheme has a lower maximum than Jameson's scheme and the new scheme on the upper side of the aerofoil. The reason for this stems from the fact that Roe's dissipation model is more dissipative than Jameson's and the new scheme.

The lift and drag coefficients computed for the steady-state solution for the three separate dissipation models are as shown in Table III. It can be seen that the coefficients for Jameson's 


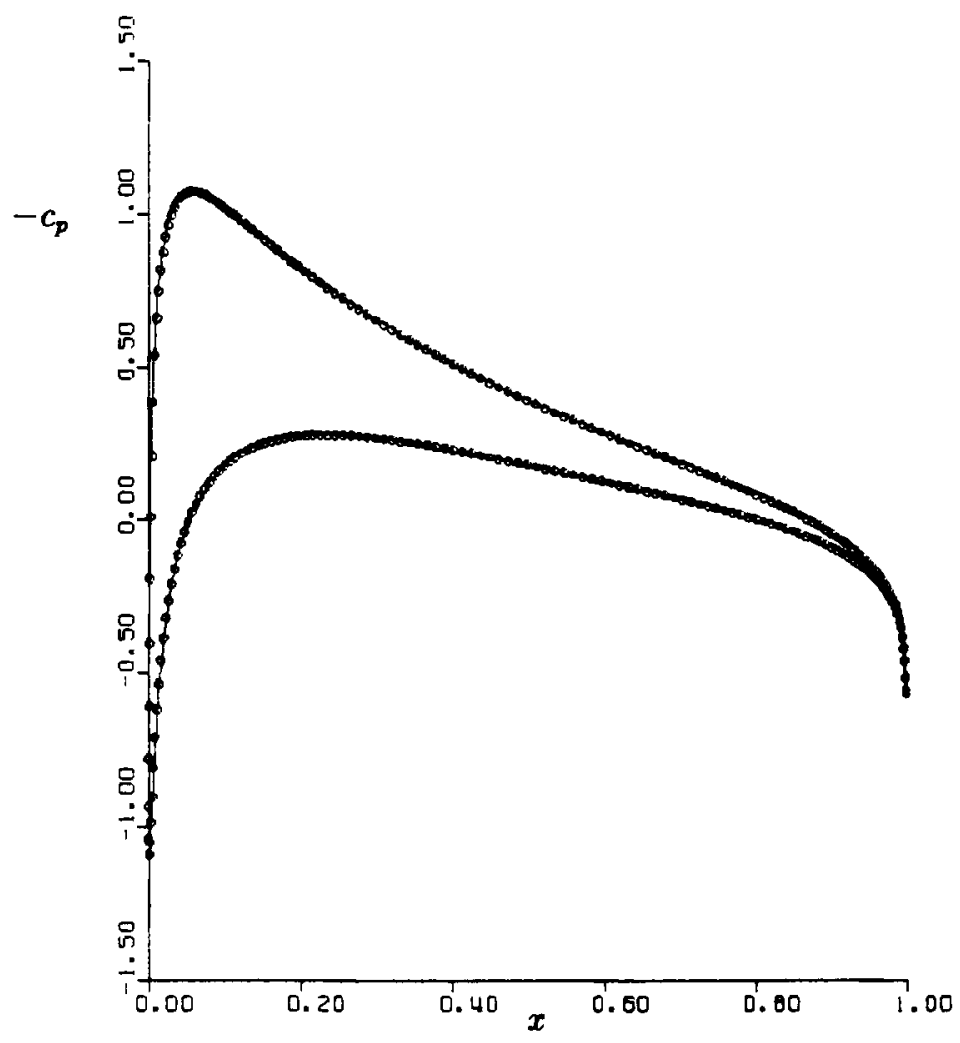

Figure 4. The pressure coefficient $c_{\mathrm{p}}$ along the surface $x$ of the aerofoil for $M_{\infty}=0.63$ and $\alpha=2^{\circ}$ for Jameson's dissipation model on the $257 \times 129$ grid

scheme and the new scheme are approximately the same. Roe's scheme results in a smaller lift coefficient and a larger drag coefficient. So, this numerical result also indicates that Roe's scheme is too dissipative. The calculated lift and drag coefficients are close to the values presented in the literature. ${ }^{12-15}$

\subsection{The second test case with $M_{\infty}=0 \cdot 8$ and $\alpha=1 \cdot 25^{\circ}$}

This test case is widely used for inviscid aerofoil computations. ${ }^{2,8,12-15}$ With this combination of free-stream Mach number and angle of attack the flow is transonic. The solution has a weak shock on the lower surface of the aerofoil, a strong shock on the upper surface of the aerofoil and a weak contact discontinuity in the wake.

The parameter settings used for this test case are also those shown in Table II. A requirement for the choice of the parameters $\kappa^{(2)}, \kappa^{(4)}, \alpha^{(4)}$ and $\delta$ was that the strong shock at the upper side of the aerofoil and the weak shock at the lower side of the aerofoil did not result in overshoots near these shocks. The pressure distributions along the aerofoil surface computed with Jameson's scheme and the new scheme are shown in Figures 6 and 7, respectively It is clearly visible that the new scheme captured the weak and the strong shock with only one interior grid point, whereas Jameson's scheme needed three interior grid points Moreover, the strong shock does not possess any wiggles However, the weak shock on the lower side of the aerofoil surface appears to have a very small overshoot. In the pressure distributions presented in Reference 14, this phenomena 


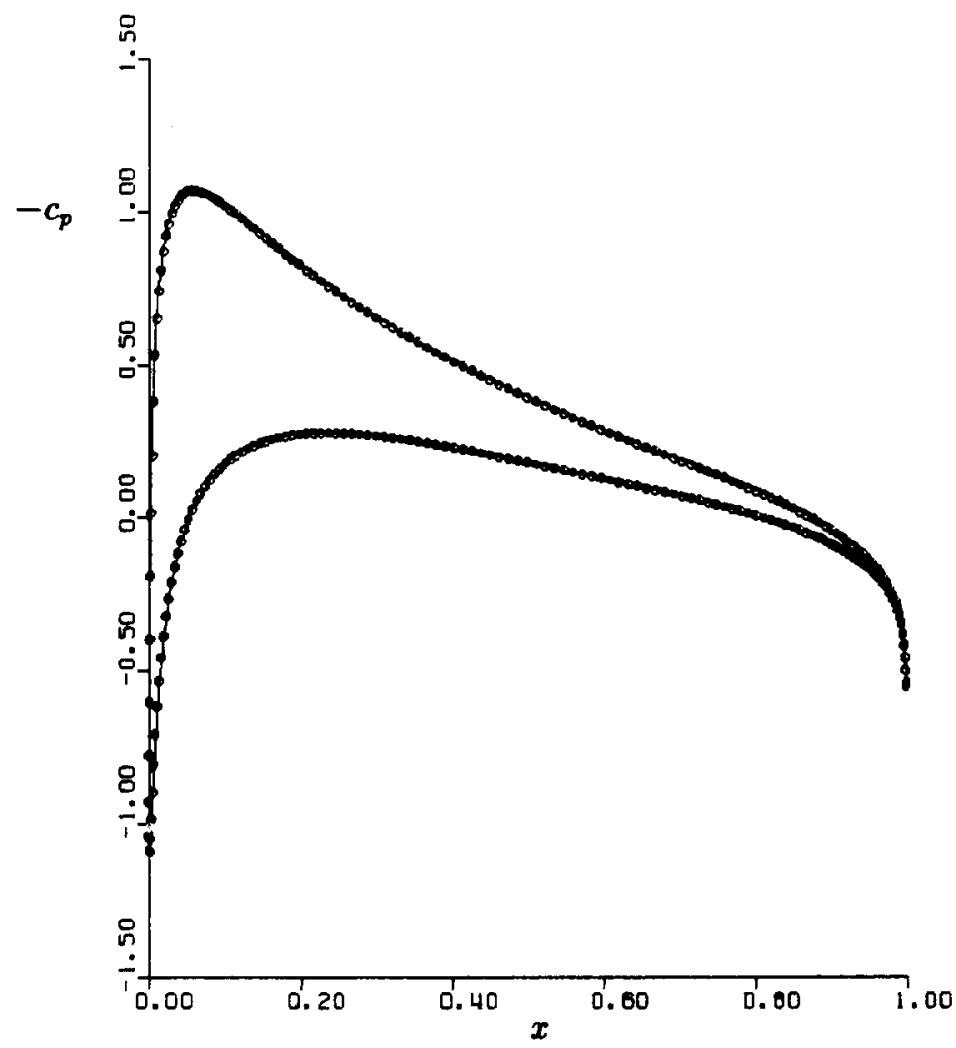

Figure 5. The pressure coefficient $c_{p}$ along the surface $x$ of the aerofoil for $M_{\infty}=0.63$ and $\alpha=2^{\circ}$ for the new dissipation model on the $257 \times 129$ grid

Table III. The lift and drag coefficients for the test case $M_{\infty}=0.63$ and $\alpha=2^{\circ}$

\begin{tabular}{lcc}
\hline Dissipation model & Lift coefficient & Drag coefficient \\
\hline Roe & 0.2937 & 0.0115 \\
Jameson & 0.3292 & 0.0005 \\
New & 0.3283 & 0.0004 \\
\hline
\end{tabular}

cannot be observed. This may be explained by the fact that a coarser grid, $128 \times 64$, was used in these experiments. Figure 8 shows the pressure distribution along the aerofoil computed with the new scheme on the $513 \times 257$ grid. In this solution the small overshoot at the weak shock can also be noticed. So, it seems that the overshoot should be present in the solution for physical reasons.

In the pressure distribution (see Figure 7) computed with the new scheme, the Zierep discontinuity ${ }^{16}$ can clearly be seen at the weak shock. A comparison with the pressure distribution produced by Jameson's scheme shows that the Zierep discontinuity is resolved more accurately by the new scheme. Jameson's scheme smeared the Zierep discontinuity.

The lift and drag coefficients shown in Table IV are practically the same for Jameson's and the new scheme. 


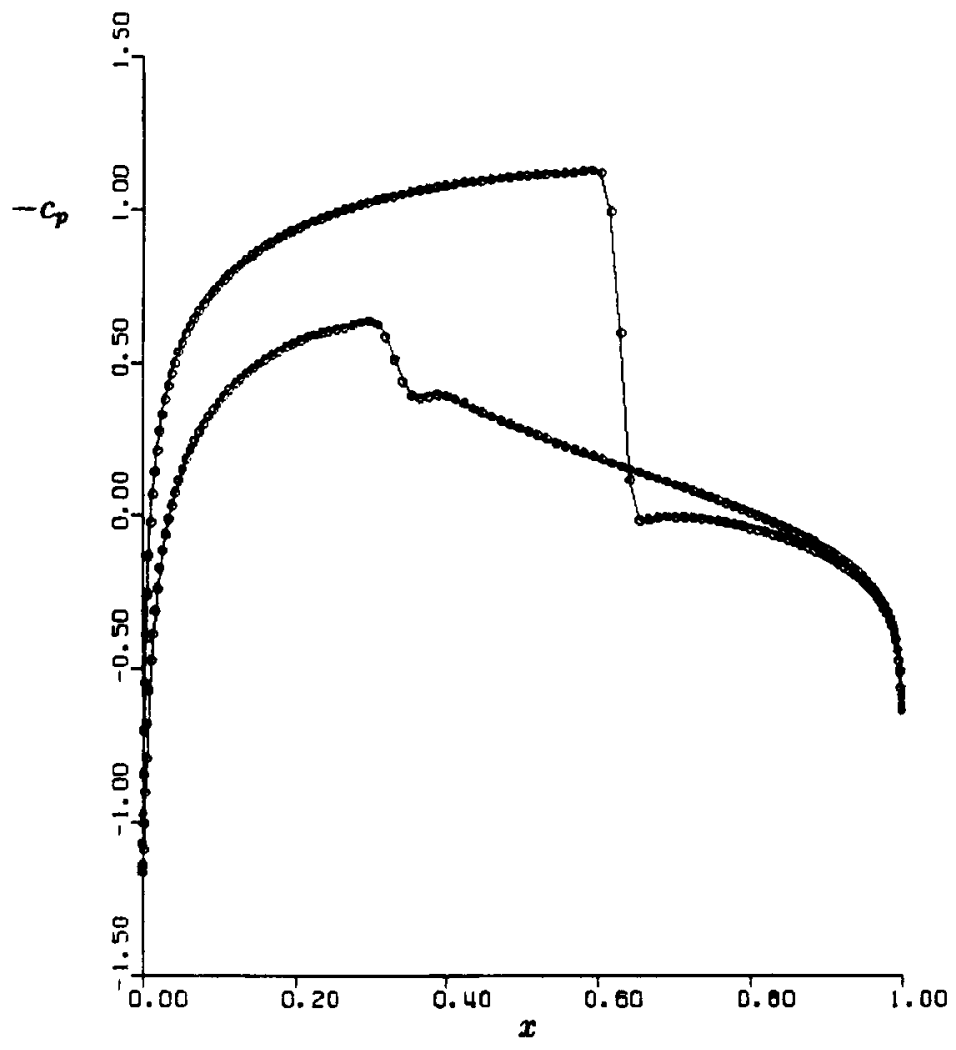

Figure 6. The pressure coefficient $c_{p}$ along the surface $x$ of the aerofoil for $M_{\infty}=0.8$ and $\alpha=1.25^{\circ}$ for Jameson's dissipation model on the $257 \times 129$ grid

The improvement in the shock-capturing can also be seen in Figures 9 and 10, in which contour plots of the pressure are depicted. These figures also show that for both schemes the rest of the flow field is roughly the same. The strong shock can also be noticed in the contour plots of the total pressure in Figures 11 and 12. Behind the trailing edge the contour lines spread out in the flow direction. The spreading of contour lines can also be noticed in the numerical results of van den Berg and Boerstoel, ${ }^{8}$ where an O-type grid was used. In Reference 12 a C-type grid was used for the same situation and the effect did not occur. The spreading of the contour lines can be explained by the irregular shape of the computational cells at the trailing edge (see Figure 13). This leads to large discretization errors. Firstly, the cell aspect ratio of the cells at the trailing edge is approximately equal to 2 , and secondly, the cells at the trailing edge possess acute and obtuse angles. In a $\mathrm{C}$-type grid the cells at the trailing edge are well-shaped, implying that large discretization errors do not occur. The convergence histories for Jameson's scheme and the new scheme are shown in Figures 14 and 15, respectively. These figures show that the convergence rate of the new scheme is almost equal to the convergence rate of Jameson's scheme. The experiments were performed on a CONVEX 220 computer. The calculation time needed for $100 \mathrm{~V}$-cycles was approximately $106 \mathrm{~min}$ for the new scheme, whereas it was $87 \mathrm{~min}$ for Jameson's scheme. 


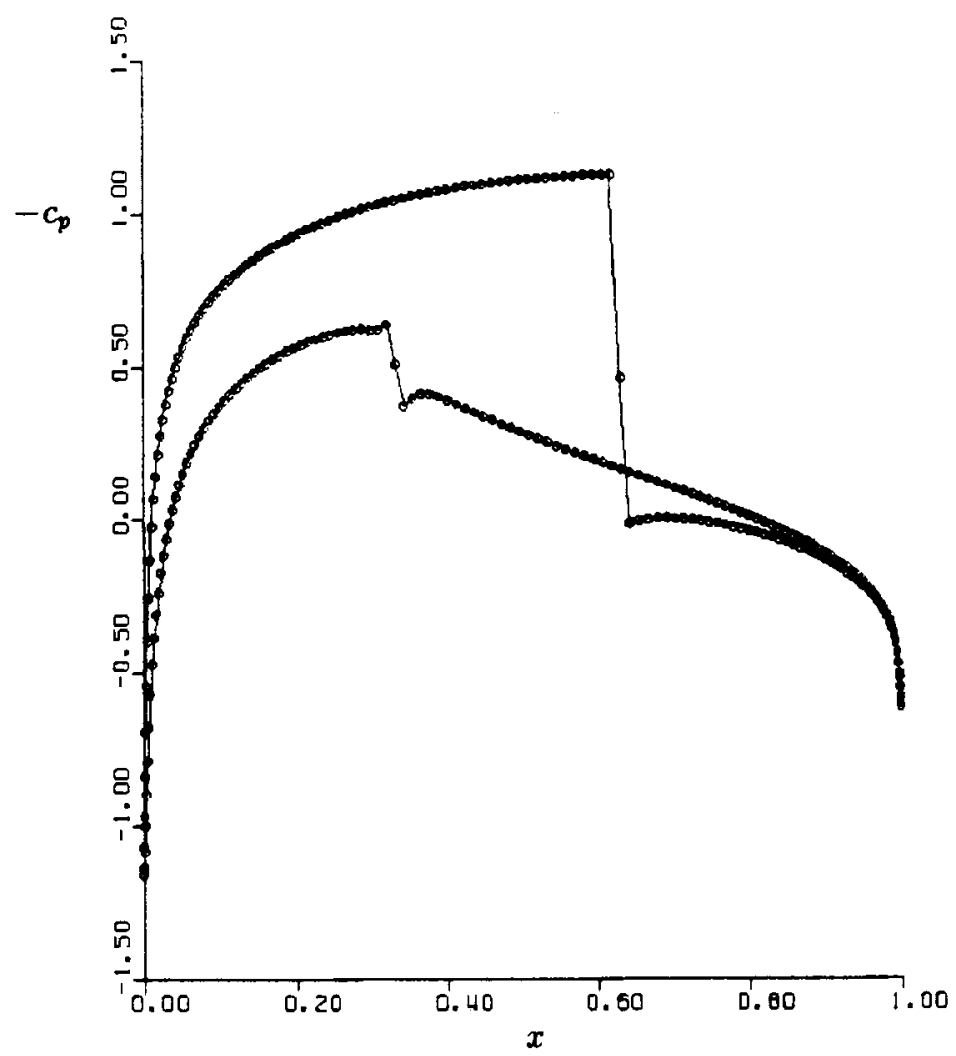

Figure 7. The pressure coefficient $c_{p}$ along the surface $x$ of the aerofoil for $M_{\infty}=0.8$ and $\alpha=1.25^{\circ}$ for the new dissipation model on the $257 \times 129$ grid

\subsection{Grid refinement study}

In order to examine whether the discrete steady-state solution converges to a limit solution when the grid is refined, a grid refinement study is carried out for the new scheme. Due to the fact that the exact solution for this model problem is unknown, the grid refinement study is restricted to the investigation of the convergence behaviour of the lift and the drag coefficient. For the new scheme it is to be expected that the lift and the drag coefficients converge to a limit solution with second-order accuracy in space.

In the grid refinement study the transonic test case $M_{\infty}=0.8$ and $\alpha=125^{\circ}$ is used where the parameters are chosen according to Table II. Two grid sequences are used to examine the convergence behaviour of the lift and the drag coefficients. The coarse grids are obtained from the finest grid by performing subsequent injections. The results obtained are shown in Table V. The lift coefficients do not show a monotonic behaviour when the grid is refined. For this reason one cannot easily employ an error analysis for this quantity. Finer grids are necessary to investigate the behaviour of the lift coefficient on grid refinement.

In contrast, the drag coefficient does show a monotonic behaviour. So, assume that the drag coefficient is a function of the grid size $h$ in the following way:

$$
c_{\mathrm{d}}(h)=A+B h^{C}
$$




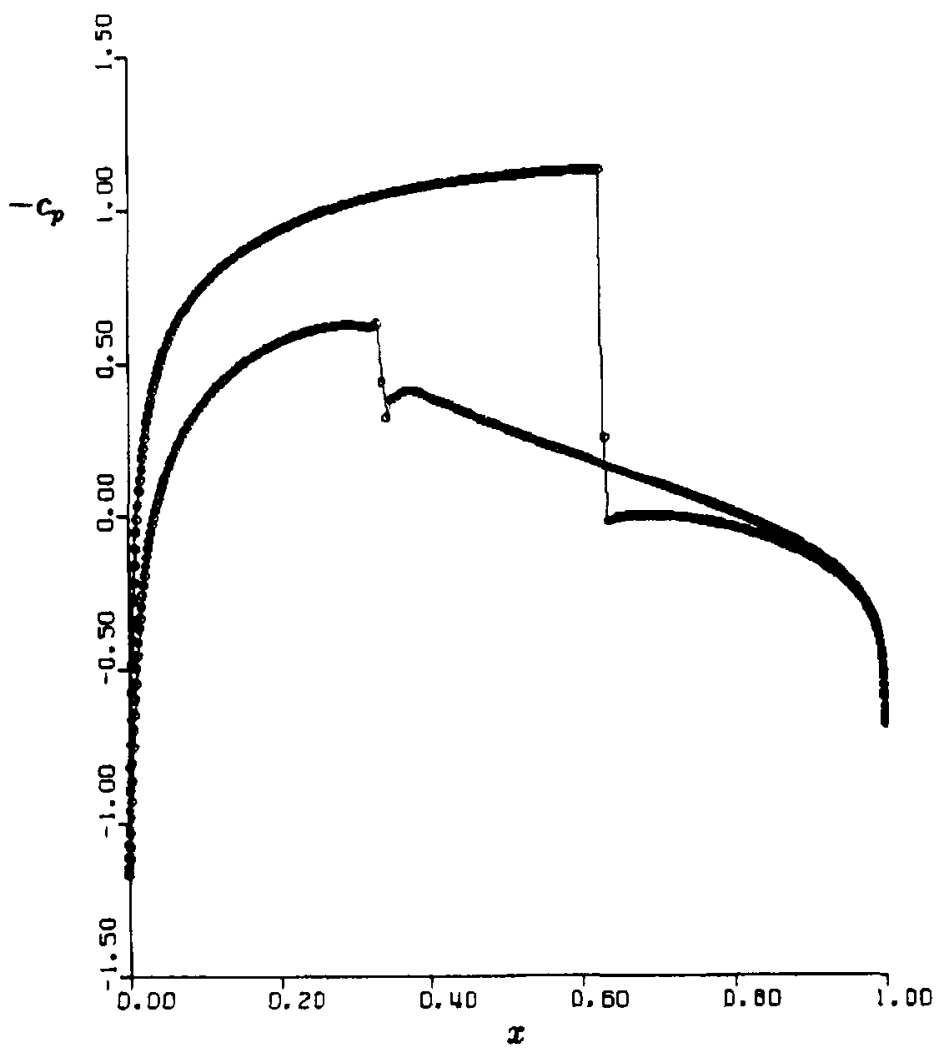

Figure 8. The pressure coefficient $c_{\mathrm{p}}$ along the surface $x$ of the aerofoil for $M_{\infty}=0.8$ and $\alpha=1.25^{\circ}$ for the new dissipation model on the $513 \times 257$ grid

Table IV. The lift and drag coefficients for the test case $M_{\infty}=0.8$ and $\alpha=1.25^{\circ}$

\begin{tabular}{lcc}
\hline Dissipation model & Lift coefficient & Drag coefficient \\
\hline Jameson & 0.3458 & 0.0222 \\
New & 0.3455 & 0.0221 \\
\hline
\end{tabular}

where $A, B$ and $C$ are constants. The quantity $A$ represents the limit value of the drag coefficient for $h=0$ and the constant $C$ denotes the order of accuracy of the space discretization used. These constants can be determined exactly from the drag coefficients shown in Table $\mathrm{V}$ by using the drag coefficient of three subsequent grids. The constants obtained in this way are shown in Table VI.

This table shows that the limit value of the drag coefficient is equal to $A=0.02188$. The limit value for the order of accuracy of the space discretization is approximately equal to $C=2 \cdot 0$, which corresponds with the theoretical order of accuracy for the new scheme. 


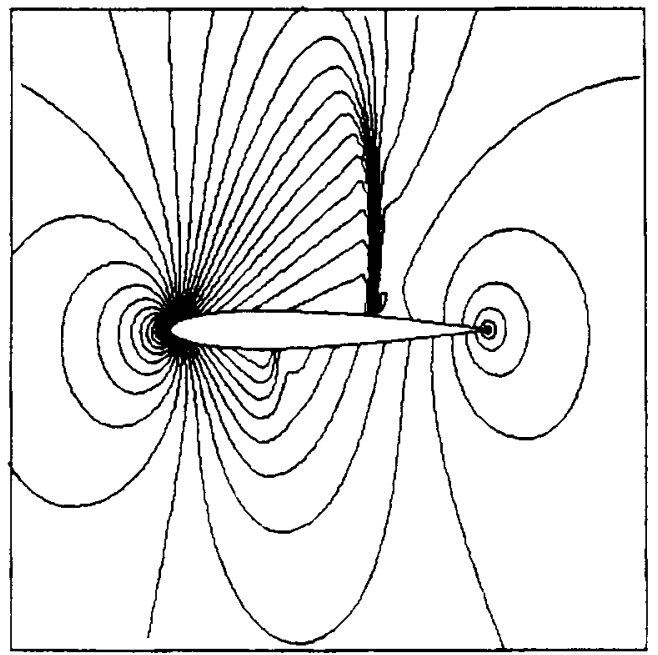

Figure 9. Contour plot of the pressure for $M_{\infty}=0.8$ and $\alpha=1.25^{\circ}$ for Jameson's dissipation model on the $257 \times 129$ grid

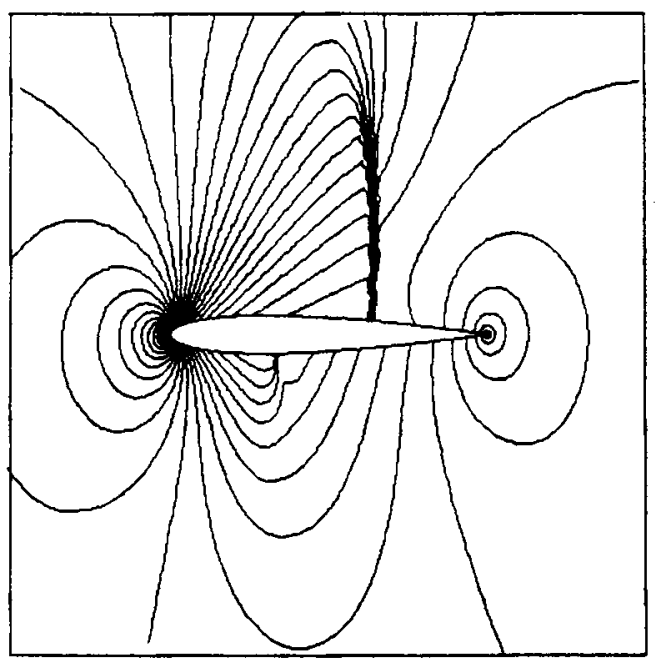

Figure 10. Contour plot of the pressure for $M_{\infty}=0.8$ and $\alpha=1.25^{\circ}$ for the new dissipation model on the $257 \times 129$ grid. The contour lines drawn in this picture and the one before are the same

\section{CONCLUSION}

Comparison of numerical results for Jameson's scheme and the new scheme shows that the shock-capturing of the new scheme is more accurate than the shock-capturing of Jameson's scheme. For both test cases the new scheme results in approximately the same lift and drag coefficients as Jameson's scheme. Moreover, the convergence rates of both schemes agree quite well. Due to the fact that Roe's dissipative flux is used in the new scheme, an entropy fix becomes necessary. 


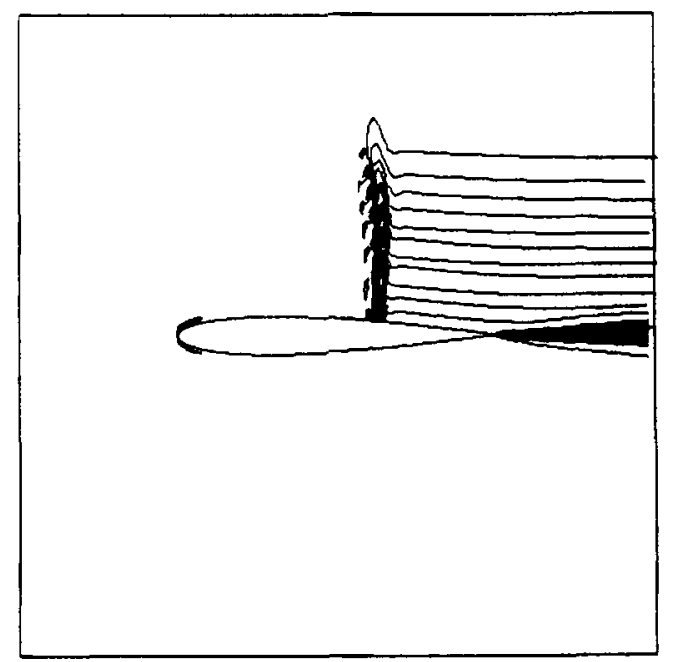

Figure 11. Contour plot of the total pressure $p_{\mathrm{t}}$ for $M_{\infty}=0.8$ and $\alpha=1.25^{\circ}$ for Jameson's dissipation model on the $257 \times 129$ grid

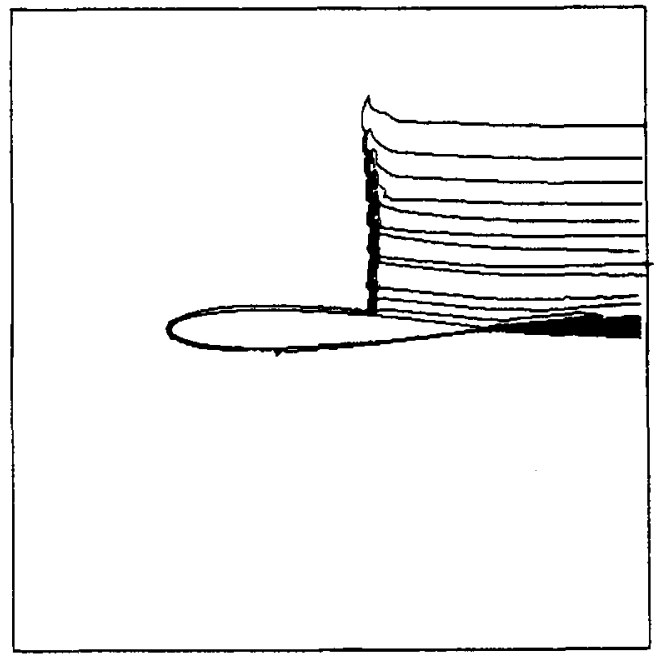

Figure 12. Contour plot of the total pressure $p_{1}$ for $M_{\infty}=0.8$ and $\alpha=1.25^{\circ}$ for the new dissipation model on the $257 \times 129$ grid

From the grid refinement study, it follows that the order of accuracy of the space discretization is approximately equal to two. This corresponds to the theoretical accuracy of the space discretization of the new scheme.

The main drawback of the replacement of Lax-Friedrichs' flux in Jameson's scheme with Roe's dissipative flux is that the calculation time increases by $20 \%$. This increase is acceptable, because the total computer time needed in order to reach the steady-state solution is relatively low. 


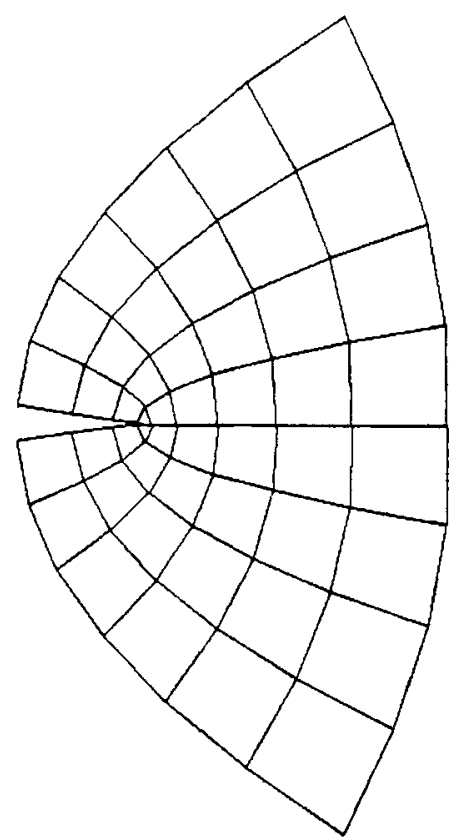

Figure 13. An enlarged view of the grid at the trailing edge

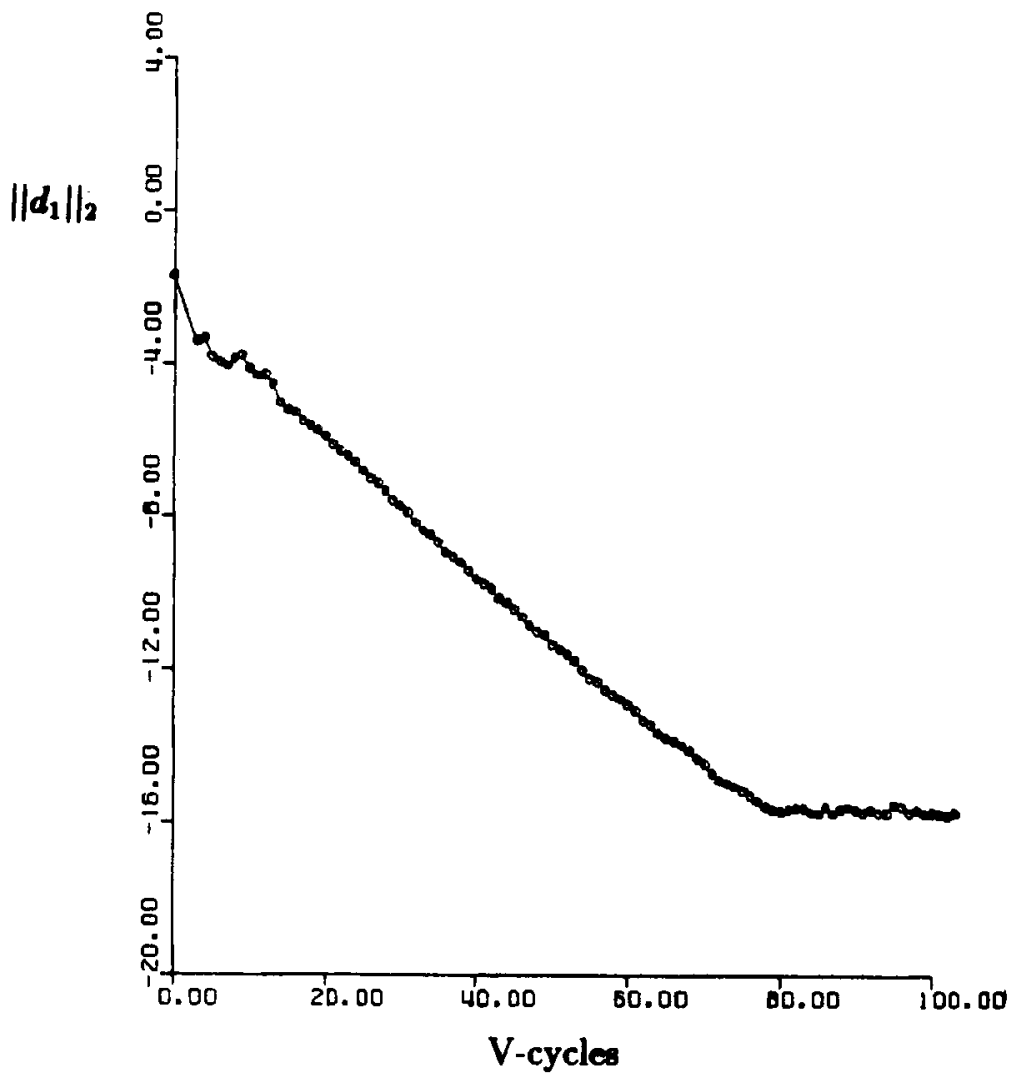

Figure 14. Convergence history for $M_{\infty}=0.8$ and $\alpha=1.25^{\circ}$ for Jameson's dissipation model on the $257 \times 129$ grid 


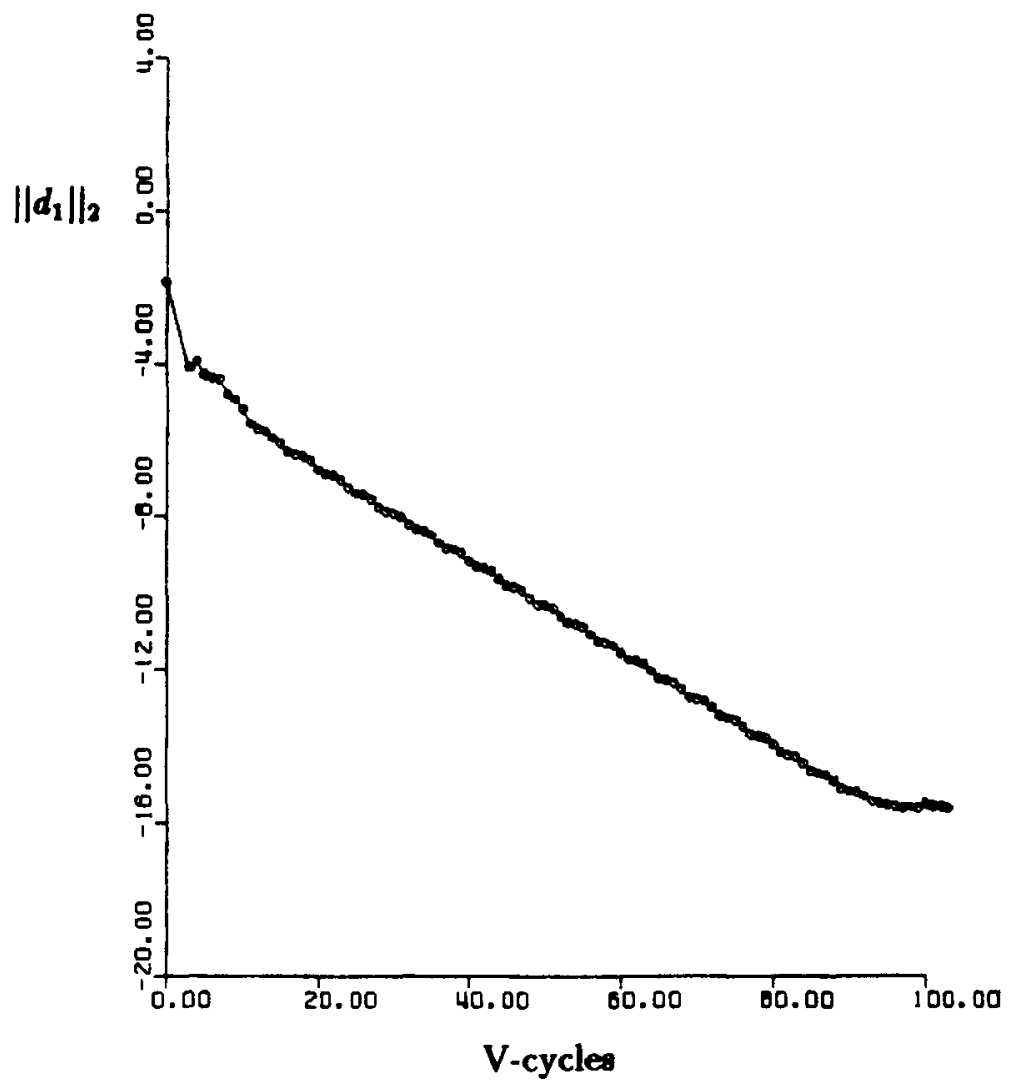

Figure 15. Convergence history for $M_{\infty}=0.8$ and $\alpha=1.25^{\circ}$ for the new dissipation model on the $257 \times 129$ grid

Table V. The lift coefficient $c_{1}$, and the drag coefficients, $c_{\mathrm{d}}$, as functions of the grid size $h$

\begin{tabular}{lccc}
\hline Grid & Grid size, $h$ & $c_{1}$ & $c_{\mathbf{d}}$ \\
\hline $65 \times 33$ & 1 & 0.339537 & 0.0278144 \\
$129 \times 65$ & $1 / 2$ & 0.346178 & 0.0227719 \\
$257 \times 129$ & $1 / 4$ & 0.345231 & 0.0220810 \\
$513 \times 257$ & $1 / 8$ & 0.343783 & 0.0219266 \\
$49 \times 25$ & 1 & 0.324865 & 0.0347127 \\
$97 \times 49$ & $1 / 2$ & 0.346084 & 0.0237998 \\
$193 \times 97$ & $1 / 4$ & 0.347023 & 0.0222952 \\
$385 \times 193$ & $1 / 8$ & 0.344289 & 0.0219690 \\
\hline
\end{tabular}

Table VI. The constants $A, B$ and $C$ at different grids

\begin{tabular}{lccc}
\hline Grid & $A$ & $B$ & $C$ \\
\hline $129 \times 65$ & 0.02197 & $5.84 \times 10^{-3}$ & 2.87 \\
$257 \times 129$ & 0.02188 & $8.89 \times 10^{-4}$ & 2.16 \\
$97 \times 49$ & 0.02205 & $1.26 \times 10^{-2}$ & 2.86 \\
$193 \times 97$ & 0.02188 & $1.92 \times 10^{-3}$ & 2.21 \\
\hline
\end{tabular}


So, the overall conclusion is that the shock-capturing of Jameson's scheme can be improved at the expense of a moderate increase of the calculation time.

\section{REFERENCES}

1. P. L. Roe, 'Approximate Riemann solvers, parameter vectors and difference schemes', J. Comput. Phys., 43, 357-372 (1981).

2. A. Jameson, 'Transonic flow calculations', MAE-report 1651, Princeton University, 1983.

3. P. D. Lax, 'Shock waves and entropy', in E. H. Zarantello (ed.), Contributions to Nonlinear Functional Analysis, Academic Press, New York, 1971, pp. 603-634.

4. A. Harten, 'On a class of high resolution total-variation-stable finite-difference schemes', SIAM J. Numer. Anal., 21, 1-23 (1984).

5. E. Dick, 'Second-order formulation of a multigrid method for steady Euler equations through defect-correction', J. Comput. and Appl. Math., 35, 159-168 (1991).

6. S. Osher and S. C. Chakravarthy, 'Upwind schemes and boundary conditions with applications to Euler equations in general geometries', J. Comput. Phys., 50, 447-481 (1983).

7. R. Courant and K. O. Friedrichs, 'Supersonic flow and Shock Waves', Interscience, New York, 1948.

8. J. I. van den Berg and J. W. Boerstoel, 'Theoretical and numerical investigation of characteristic boundary conditions for cell-centered Euler flow calculations', $N L R$ TR $88124 L, 1988$.

9. R. Radespiel, 'A cell-vertex multigrid method for the Navier-Stokes equations', NASA Techn. Mem. 101557, 1989.

10. J. W. van der Burg, J. G. M. Kuerten and P. J. Zandbergen, 'Multigrid and Runge-Kutta timestepping applied to the uniformly non-oscillatory scheme for conservation laws', J. of Eng. Math., 25, 243-263 (1991).

11. L. Martinelli, 'Calculations of viscous flows with a multigrid method', Ph.D. Dissertation, MAE Department, Princeton University, 1987.

12. F. J. Brandsma, 'Validation testplan for single aerofoils', NLR TP 89228 L, 1989.

13. F. J. Brandsma, 'Validation report of the ISNaS single-block Euler pilot code', NLR TP $90242 L, 1990$.

14. B. Koren, 'Multigrid and defect correction for the steady Navier-Stokes equations', Ph.D. Dissertation, Centre for Mathematics and Computer Science, The Netherlands, 1989.

15. A. Rizzi and H. Viviand, 'Numerical methods for the computation of inviscid transonic flows with shock waves', A GAMM Workshop, Notes on Numerical Analysis, 3, 167-221 (1981).

16. J. Zierep, 'Der senkrechte verdichtungsstoss am gekrümmten profil', Z. Angew. Math. Phys., 9b, 764-776 (1958). 